\title{
Inkjet Printed Planar Coil Antenna Analysis for NFC Technology Applications
}

\author{
I. Ortego, ${ }^{1}$ N. Sanchez, ${ }^{1}$ J. Garcia, ${ }^{1}$ F. Casado, ${ }^{2}$ D. Valderas, ${ }^{1}$ and J. I. Sancho' \\ ${ }^{1}$ Electronics and Communications Department, CEIT and Tecnun University of Navarra, Manuel de Lardizábal 15, \\ 20018 San Sebastián, Spain \\ ${ }^{2}$ Communications Area, IKERLAN-IK4, P J.M. Arizmendiarrieta, 20500 Arrasate-Mondragon, Spain
}

Correspondence should be addressed to I. Ortego, iortego@ceit.es

Received 15 December 2011; Accepted 9 January 2012

Academic Editor: Ana Collado

Copyright (C) 2012 I. Ortego et al. This is an open access article distributed under the Creative Commons Attribution License, which permits unrestricted use, distribution, and reproduction in any medium, provided the original work is properly cited.

\begin{abstract}
The aim of this paper is to examine the potential of inkjet printing technology for the fabrication of Near Field Communication (NFC) coil antennas. As inkjet printing technology enables deposition of a different number of layers, an accurate adjustment of the printed conductive tracks thickness is possible. As a consequence, input resistance and $Q$ factor can be finely tuned as long as skin depth is not surpassed while keeping the same inductance levels. This allows the removal of the typical damping resistance present in current NFC inductors. A general methodology including design, simulation, fabrication, and measurement is presented for rectangular, planar-spiral inductors working at $13.56 \mathrm{MHz}$. Analytical formulas, computed numerical models, and measured results for antenna input impedance are compared. Reflection coefficient is designated as a figure of merit to analyze the correlation among them, which is found to be below $-10 \mathrm{~dB}$. The obtained results demonstrate the suitability of this technology in the fabrication of low cost, environmentally friendly NFC coils on flexible substrates.
\end{abstract}

\section{Introduction}

Near Field Communication (NFC) is a recent wireless technology working on HF band that enables the exchange of data in a short range. It is an ideal solution where reliable exchange of data is paramount to quality user operations such as mobile payments. It is estimated that this system will be used in more than 1.2 billion smartphones by 2015 [1]. Therefore, it is necessary to reduce the cost of the manufacturing process keeping at the same time an environmentally friendly approach to pave the way for this promising technology.

In this context, there are a number of approaches to implement an NFC antenna layout onto a selected substrate. The earliest and most cumbersome method of antenna fabrication has been subtractive etching on a metalplated laminate (e.g., FR4). Subtractive etching has matured to the point where it has become a low-tech process requiring inexpensive off-the-shelf materials and equipment. However, for larger production runs, etching creates signif- icant amounts of metal salt and chemical waste products, incurring increased costs due to regulatory fees.

Contrary to the traditional etching technique, inkjet printing technology is a direct-write technology by which the designed pattern is transferred directly to the substrate [2]. This technology has become a major topic in scientific research, especially drop-on-demand (DOD) inkjet printing systems [3]. With these systems, the production costs of electronic devices are dramatically reduced, as material can be deposited on demand.

It has been estimated that there are about 1500 worldwide research units working on various aspects of flexible electronics [4]. The fabrication of thin conductive tracks by methods of inkjet printing has been recently investigated extensively $[5,6]$.

Up to date, inks made of silver conductive nanoparticles have been used to produce printed electronic circuits on flexible surfaces. Metallic nanoparticle inks typically consist of silver or gold nanoparticles with diameters ranging from just $2 \mathrm{~nm}$ to over $50 \mathrm{~nm}$, encapsulated by a protective shell 
and dispersed in a liquid solvent $[7,8]$. Once printed, a continuous conducting structure can be obtained by sintering, that is, by increasing the temperature of the structure so that the separate nanoparticles melt together.

In the fabrication process, several parameters are involved, such as, size of ink nanoparticles, substrate, surface tension of the particle and of the substrate, time and temperature of the ink sintering, among others [9], which provide a wide range of degree of freedom for circuit designs, and particularly for antennas, on flexible substrates. Future terminals, such as smartphones, are envisaged to be completely flexible in the near future [10-12]. The antenna has a considerable size in the system; therefore, its flexibility is crucial if a flexible terminal is desired.

The usage of inkjet technology for NFC implementation on flexible terminals stills finds another motivation for the antennas involved. The $Q$ factor required for the antenna is directly related to the read range to be achieved by the technology. For example, in a typical RFID system, for a distance of $38 \mathrm{~cm}, Q$ factors close to 40 are required $[13,14]$. As NFC allows for shorter distances motivated for a whole sort of reasons, as, for example, security and privacy of users, restrictions on tag $Q$ factors are relaxed to get the same voltage to energize the RFID tag integrated circuit.

As long as a decreasing of $Q$ factor can be tolerated by performing antennas with tracks thinner than their skin depth, the input resistance can be increased to make up a possible removal of the damping resistance. With copperetched antennas, $Q$-factors of more than 100 could be realized $[13,15]$. In this technology, the conductive track thickness is fixed and longer than the skin depth of the copper $(18 \mu \mathrm{m}$ at $13.56 \mathrm{MHz})$. However, inkjet printing technology allows to vary the thickness of the printed layers with approximately $1 \mu \mathrm{m}$ resolution all the way from $0 \mu \mathrm{m}$ to the skin depth. Thus, typical coil antenna manufactured by inkjet printing technology exhibits small $Q$ factors of around 4-10 [5].

Therefore, an exploration of the potentials and limitations of inkjet printing technology for NFC flexible terminals should be addressed in this context since it provides a low cost, environmentally friendly manufacturing process on flexible materials with no damping resistance involved.

This paper provides a rigorous approach, involving analytical and computational models, for rectangular planar spiral coils at $13,56 \mathrm{MHz}$ with subsequent experimental verification on flexible substrates. It is divided into four parts. The second section deals with the methodology, where a general method for the design, fabrication, simulation, and measurement of inkjet printed loop antennas for NFC systems on a flexible substrate is explained. The third part includes the implementation and obtained results. Lastly, the fourth part states the conclusions.

\section{Methodology}

2.1. Analytical Approach. In this section, all the necessary equations to analytically calculate the total inductance, $Q$ factor, and resistance of a rectangular planar spiral inductor (Figure 1) will be shown.

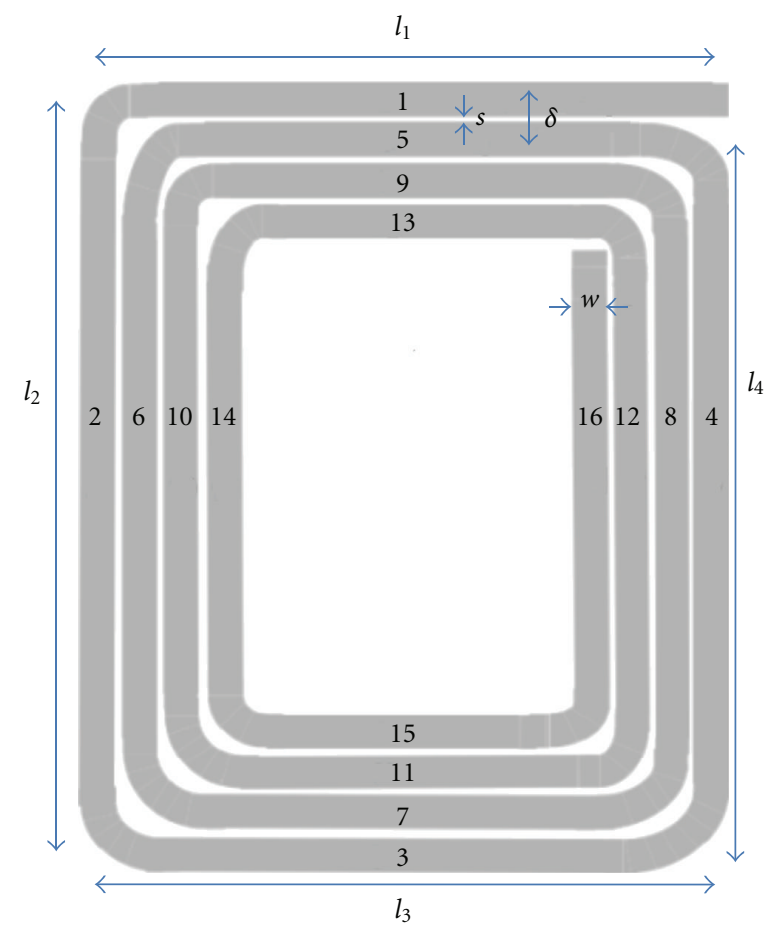

FIgURE 1: Rectangular planar spiral inductor.

2.1.1. Inductance Calculation. The total inductance of an Nturn planar spiral inductor coil (Figure 1) can be calculated as follows:

$$
L_{T}=L_{0}+M_{+}-M_{-}[\mu \mathrm{m}],
$$

where $L_{T}$ is the total inductance, $L_{0}$ is the sum of self inductances of all straight segments, $M_{+}$is the sum of positive mutual inductances, and $M_{-}$is the sum of negative ones.

The self inductances of a rectangular cross-section conductor can be obtained using the following equation [14]:

$$
L=0,002 l\left\{\ln \left(\frac{2 l}{w+t}\right)+0.50049+\frac{w+t}{3 l}\right\}[\mu \mathrm{m}],
$$

where $w, t$, and $l$ are the segment's width, thickness, and length in $\mathrm{cm}$, respectively.

The mutual inductance is the inductance that results from the magnetic fields produced by adjacent conductors. The mutual inductance is positive when the directions of the current along the conductors are in the same direction, and negative when the directions of currents are in opposite directions. The mutual inductance between two parallel conductors of equal length is a function of the length of the conductors and of the geometric mean distance $d$ between them. It is calculated by [16]

$$
M=2 l F[n H]
$$

where $F$ can be calculated as

$$
F=\ln \left\{\left(\frac{l}{d}\right)+\left[1+\left(\frac{l}{d}\right)^{2}\right]^{1 / 2}\right\}-\left[1+\left(\frac{d}{l}\right)^{2}\right]^{1 / 2}+\left(\frac{d}{l}\right) .
$$




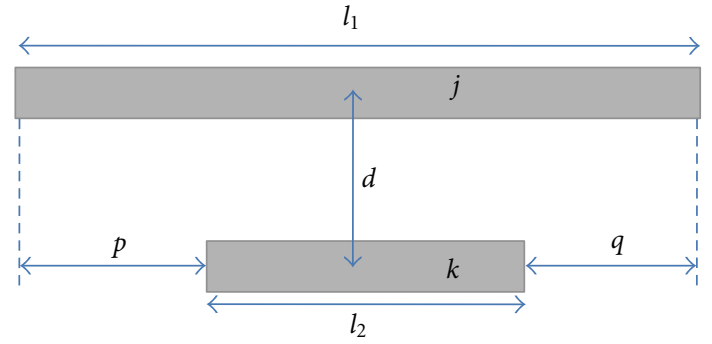

Figure 2: Two conductor segments for mutual inductance calculation.

The geometric mean distance $d$ is approximately equal to the distance between the track centres of the conductors involved. When the two segments are not equal in length, (5) should be applied according to Figure 2:

$$
M_{k, p}=\frac{1}{2}\left\{\left(M_{k+p}+M_{k+q}\right)-\left(M_{p}+M_{q}\right)\right\},
$$

where

$$
\begin{aligned}
M_{k+p}= & 2 l_{k+p} F_{k+p}, \\
F_{k+p}= & \ln \left\{\left(\frac{l_{k+p}}{d_{j, k}}\right)+\left[1+\left(\frac{l_{k+p}}{d_{j, k}}\right)^{2}\right]^{1 / 2}\right\} \\
& -\left[1+\left(\frac{d_{j, k}}{l_{k+p}}\right)^{2}\right]^{1 / 2}+\left(\frac{d_{j, k}}{l_{k+p}}\right) .
\end{aligned}
$$

2.1.2. Resistance and Quality Factor Calculation. The thickness of the conductive track deposited by inkjet printing technology can be adjusted by the number of layers micron by micron. Provided that skin effect at $13,56 \mathrm{MHz}$ is approximately $47 \mu \mathrm{m}$ for the silver conductive ink, the input resistance varies inversely with the thickness $t$ according to the following expression:

$$
R=\frac{1}{\sigma} \cdot \frac{L}{S}=\frac{1}{\sigma} \cdot \frac{L}{t \cdot w},
$$

where $R$ is the electrical resistance, $\sigma$ is the ink's conductivity, $L$ is the longitude, $S$ is the track cross section, $t$ is the thickness, and $w$ is the width of the track.

The spiral inductor $Q$ factor is then calculated as:

$$
Q=\frac{L \omega}{R}
$$

2.2. Numerical Computation. Frequency domain solver implemented by CST MICROWAVE STUDIO simulation software has been selected to make the numerical computation of the design. Adaptative hexahedral mesh is used and local mesh refinement strategy is adopted within the coil conductive track.

2.3. Implementation. The material used as substrate is Dupont Kapton polyimide film $127 \mu \mathrm{m}$ thick. It can remain stable in a wide range of temperatures, from -269 to $+400^{\circ} \mathrm{C}$.

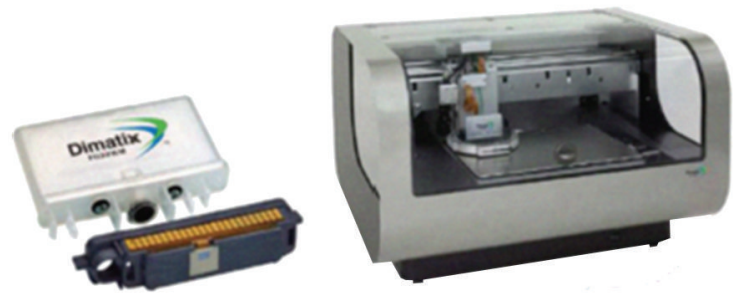

FIgure 3: Cartridge and Printer Dimatix 2831 [17].

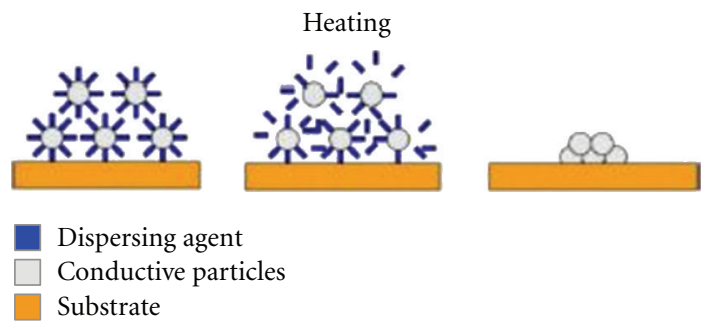

FIGURE 4: Sintering process [9].

SunTronic Jettable Silver U5714 ink by Sunjet has been used as conductive ink. It is a solvent-based silver nanoparticle inkjet ink and $20 \%$ of its weight is due to silver particles.

The printer that has been used is the Dimatix Materials Printer DMP-2831 (Figure 3). The platen temperature can be varied from $28^{\circ} \mathrm{C}$ to $60^{\circ} \mathrm{C}$. In the fabricated prototypes, $60^{\circ} \mathrm{C}$ is chosen as it prevents the ink from spreading out.

DMC-11610 cartridge which has 16 nozzles, $254 \mu \mathrm{m}$ spaced with a nominal opening of $21 \mu \mathrm{m}$, and a $10 \mathrm{pL}$ drop volume capacity has been employed. Only two nozzles with $16-17 \mathrm{v}$ applied voltage, $40^{\circ} \mathrm{C}$ of temperature and a meniscus vacuum of 4,5 inches $\mathrm{H}_{2} \mathrm{O}$ have been used at a height of $0,5 \mathrm{~mm}$ with a drop spacing of $20 \mu \mathrm{m}$.

Once the prototypes have been printed, a sintering process is required to improve the conductivity of the ink. The manufacturer recommends a range of temperature and time to sinter the silver ink and this depends on the amount of solid material present in the ink. In general, the lower the sintering time or temperature, the higher the electric resistance since a larger gap between the nanoparticles occurs. This process (Figure 4) determines the final performance of the ink. A sintering process of $30 \mathrm{~min} @ 200^{\circ} \mathrm{C}$ has been selected for all the prototypes.

\subsection{Measurements}

2.4.1. Thickness of Printed Traces. Once the methodology to print is defined, some traces are printed into a substrate to study the corresponding thickness depending on the number of layers.

A KLA-Tencor P-16+ Profiler has been used to measure the overall thickness of the printed traces and estimate the one of each layer by average. In Figure 5, the obtained profile of the printed traces is shown for different number of layers. 


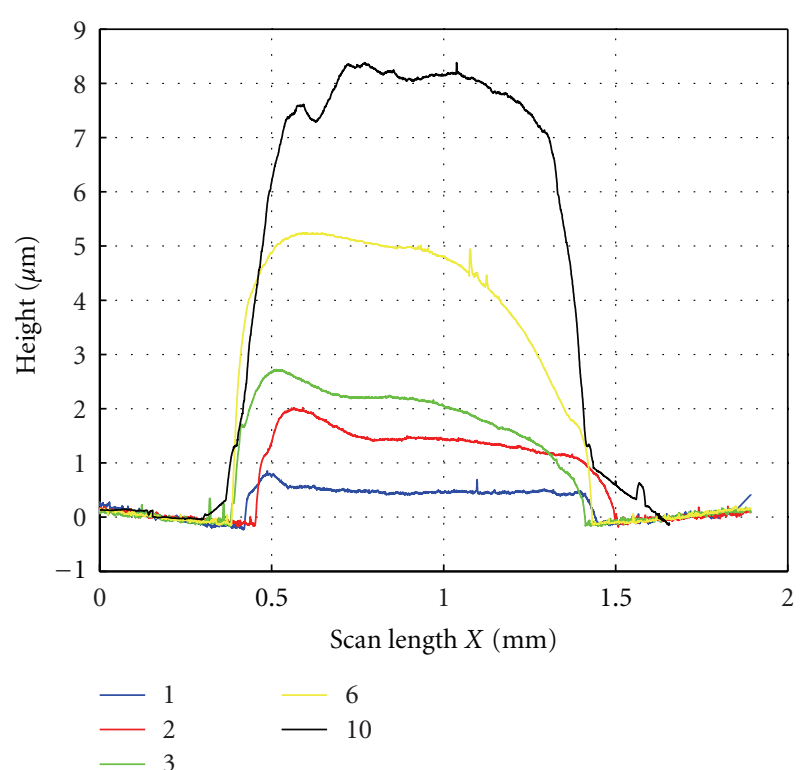

FIGURE 5: Obtained profiles for different number of printed layers.

TABLE 1: Obtained conductivity values with 30 min@200 $\mathrm{C}$ sintering process.

\begin{tabular}{lcc}
\hline Material: silver & $\begin{array}{c}\text { Conductivity } \\
(\Omega \mathrm{m})^{-1}\end{array}$ & $\begin{array}{c}\text { Conductivity } \\
\text { with respect to } \\
\text { pure silver }(\%)\end{array}$ \\
\hline Pure & $63.01 \cdot 10^{6}$ & 100 \\
One layer & $8 \cdot 10^{6}$ & 12.6 \\
Two layers & $9 \cdot 10^{6}$ & 14.3 \\
Three layers & $7.9 \cdot 10^{6}$ & 12.5 \\
Six layers & $8.5 \cdot 10^{6}$ & 13.5 \\
\hline
\end{tabular}

A thickness of $0.8 \mu \mathrm{m}$ for each layer can be assumed when considering the obtained profiles and the measured thickness.

2.4.2. Characterization of the Conductivity of the Ink. Once the thickness of the printed layers is found, conductivity is characterized.

Although conductivity is independent of the number of printed layers, the conductivity study has been done in samples with different number of layers, which correspond to different electrical resistance and thickness pairs for comparison purposes. The electrical resistance of the structure has been measured using a Keithley 6517B Electrometer/High Resistance Meter.

The conductivity can be calculated using (7). Table 1 shows averaged results collected from various samples.

The obtained results by this method are in accordance with the ones found in bibliography $[9,18]$. As an indicative value, a $10 \%$ or $20 \%$ of the conductivity of the pure silver can be considered. In this work, a conductivity of $8 \cdot 10^{6}(\Omega \mathrm{m})^{-1}$ has been considered as representative of ink performance.

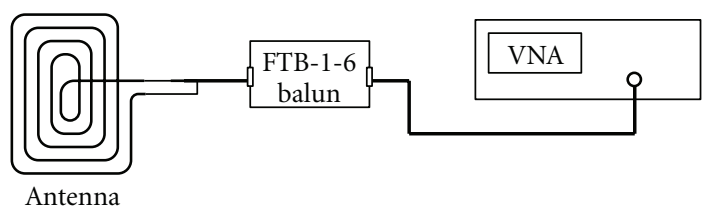

Figure 6: Measurement set up.

2.4.3. Input Impedance and $Q$ Factor. Once the antenna has been printed, its impedance can be measured by a standard VNA according to the setup shown in Figure 6 and the corresponding $Q$ factor can be calculated. A FTB-1-6 balun by Mini-Circuits is employed between the feeding cable and the fabricated prototype. The flexibility of the substrate and the ink do not allow the use of typical soldering methods. Therefore, pad connections are implemented by conductive adhesive tape.

2.5. Methodology Validation. The reflection coefficient will be used as figure of merit to estimate the correlation between the results obtained by analytical formulas, computed numerical models, and measurements.

The amplitude of the reflection coefficient between two different complex impedances can be calculated as [19]

$$
|\Gamma|=20 \log \left(\left|\frac{Z_{1}-Z_{2}^{*}}{Z_{1}+Z_{2}}\right|\right),
$$

where $Z_{1}$ and $Z_{2}$ are the complex impedances. The methodology is based on this equation by substituting $Z_{1}$ and $Z_{2}$ for pairs chosen among analytically calculated, simulated, and measured results referred to the same coil layout. The only consideration to apply this equation is that for the correct calculation one of the impedances must be conjugated (e.g., $Z_{2}=Z_{\text {ant2 }}^{*}$ ). In that way, if $|\Gamma| \leq-10 \mathrm{~dB}$, a good correlation is achieved.

\section{Implementation and Results}

An analytical study of the effect of the thickness for a 5 turn rectangular $(80 \mathrm{~mm} \times 50 \mathrm{~mm})$ planar spiral inductor with $w=s=1 \mathrm{~mm}$ has been realized. These dimensions have been selected to fulfil the ISO7810 standard [20]. This standard defines the physical characteristics of identification cards (Table 2).

The analytically obtained inductance for the presented antenna is $2.82 \mu \mathrm{H}$ according to expressions (1)-(7), and it remains unchanged with thickness $t$. However, this parameter has a strong impact on the input impedance (as the damping resistance is changed) and therefore on the coil quality factor. The obtained results are shown in Figure 7 where expressions (7) and (8) are used.

A $Q$ value up to 90 can be theoretically achieved by this technology as long as the thickness is kept below the skin depth (47 microns). For thicknesses deeper than skin depth, input resistance and $Q$ factor is no longer adjustable by this degree of freedom. 
TABLE 2: Nominal dimensions of ID-1 card type.

\begin{tabular}{lcc}
\hline Width $(\mathrm{mm})$ & Height $(\mathrm{mm})$ & Thickness $(\mathrm{mm})$ \\
\hline 85.6 & 53.98 & 0.76
\end{tabular}

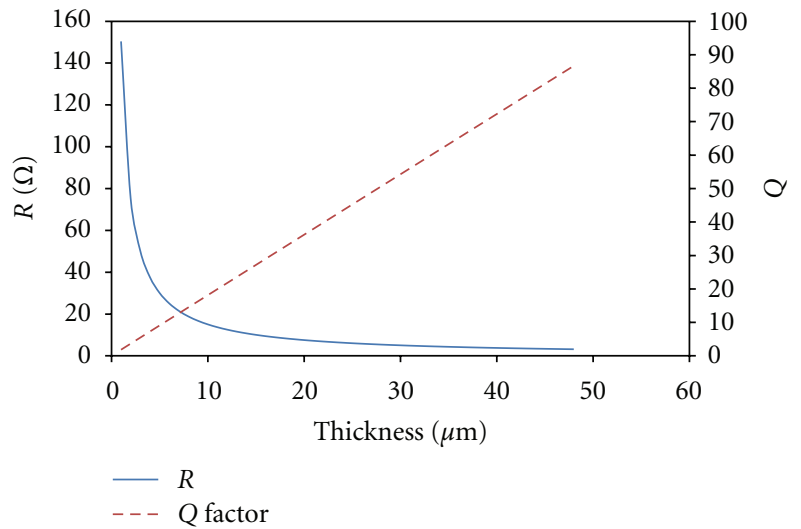

Figure 7: Calculation of $R$ and $Q$ for different thickness.

TABle 3: Obtained results of the two prototypes: analytical (A), simulation $(\mathrm{S})$, and measured $(\mathrm{M})$.

\begin{tabular}{lcccc}
\hline & & $\mathrm{A}$ & $\mathrm{S}$ & $\mathrm{M}$ \\
\hline \multirow{4}{*}{ 4 layers } & $R[\Omega]$ & 46,58 & 51,13 & 50,25 \\
& $L[\mu H]$ & 2,82 & 2,79 & 2,9 \\
& $Q$ & 5,16 & 4,64 & 4,91 \\
\hline \multirow{4}{*}{ 6 layers } & $R[\Omega]$ & 31 & 35,118 & 32,35 \\
& $L[\mu H]$ & 2,82 & 2,79 & 2,947 \\
& $Q$ & 7,75 & 6,77 & 7,76 \\
\hline
\end{tabular}

In practice, it is not feasible to fabricate coil antennas with such a high thicknesses due to the ink spilling over the track when too many layers are mounted on it. Two different prototypes have been implemented; one with 4 layers and another one with 6 layers (Figure 8) to validate the proposed methodology. The obtained results are shown in Table 3.

The correlation between the three outcomes (analytical, simulated, and measured) is studied as explained in the methodology validation section. The obtained values are summarized in Table 4.

All the correlations are below $-10 \mathrm{~dB}$; therefore, it can be concluded that the obtained results are reliable. In order to explore inkjet printing technology limits once the procedure is validated, a prototype with 10 layers has been fabricated. This represents a reasonable trade off between delivered performance in terms of $Q$ values and complexity of the manufacturing process due to the number of layers. A measured inductance value of $2.9 \mu \mathrm{H}$, a resistance of $23.5 \Omega$ and a $Q$ factor of 10.5 are obtained in perfect accordance with the aforementioned results.

\section{Conclusions}

The potential of using inkjet printing technology to fabricate NFC antennas has been investigated. A general methodology
TABLE 4: Results validation.

\begin{tabular}{lccc}
\hline \multirow{4}{*}{ 4 layers } & \multicolumn{2}{c}{ Comparison } & $|\Gamma|[\mathrm{dB}]$ \\
\hline \multirow{3}{*}{ 6 layers } & $\mathrm{A}$ & $\mathrm{S}$ & -25.16 \\
& $\mathrm{~A}$ & $\mathrm{M}$ & -21.94 \\
& $\mathrm{~S}$ & $\mathrm{M}$ & -20.35 \\
\hline & $\mathrm{A}$ & $\mathrm{S}$ & -22.78 \\
& $\mathrm{~A}$ & $\mathrm{M}$ & -15.39 \\
& $\mathrm{~S}$ & $\mathrm{M}$ & -14.08 \\
\hline
\end{tabular}

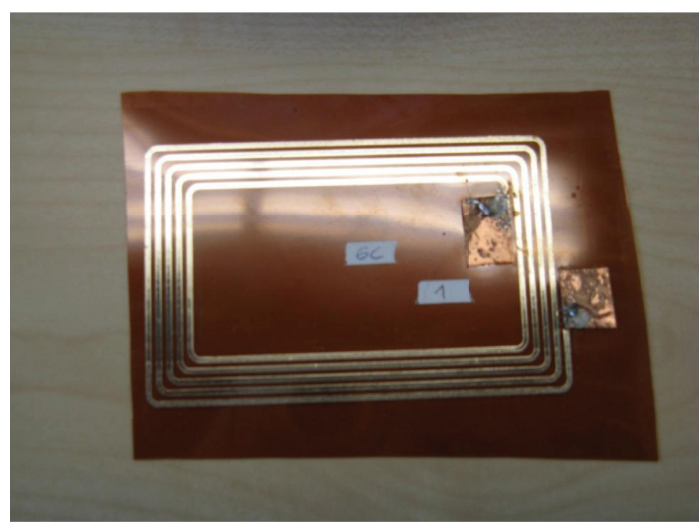

FIgURE 8: Printed prototype (6 layers).

for the design, simulation, fabrication, and measurement process has been explained.

The ink conductivity is shown to be around 8 . $10^{6}(\Omega \mathrm{m})^{-1}$ and the approximate thickness of each deposited layer $0.8 \mu \mathrm{m}$. The resistance can be controlled by the capability of this technology of varying the track thickness, which prevents from extra damping resistor implementations. With the aforementioned resolution, $Q$ values ranging from 4 to 10 can be obtained while keeping the same inductance value.

Inkjet printing technology is therefore a promising technique for the fabrication of NFC coil antennas. Moreover, due to its low cost manufacturing process and substrate flexibility, it is a perfect candidate for the implementation of environmentally friendly NFC terminals into flexible substrates.

\section{Acknowledgment}

Iñaki Ortego's scholarship has been granted by the Department of Education of the Basque Government. Joseba García's contract is partially supported by the Spanish Ministry of Education within the framework of the Torres Quevedo Program, and cofinanced by the European Social Fund. The authors would like to thank the collaboration agreement between Tecnun and CST that has made possible the achievements of this piece of research.

\section{References}

[1] "Wireless Design Online," http://www.wirelessdesignonline .com/article.mvc/NFC-Chip-Shipments-To-Surpass-12-Billio- 
n-By-000 1?sectionCode $=$ News\&templateCode $=$ SponsorHeader\&user $=1742815 \&$ source $=\mathrm{nl}: 32237$.

[2] A. Rida, R. Vyas, L. Yang, C. Kruesi, and M. M. Tentzeris, "Low cost inkjet-printing paper-based modules for RFID sensing and wireless applications," in Proceedings of the 38th European Microwave Conference (EuMC '08), pp. 1715-1718, Amsterdam, The Netherlands, October 2008.

[3] T. H. J. van Osch, J. Perelaer, A. W. M. de Laat, and U. S. Schubert, "Inkjet printing of narrow conductive tracks on untreated polymeric substrates," Advanced Materials, vol. 20, no. 2, pp. 343-345, 2008.

[4] J. Hu, "Overview of flexible electronics from ITRI's viewpoint," in Proceedings of the 28th IEEE VLSI Test Symposium (VTS '10), p. 84, Santa Cruz, Calif, USA, April 2010.

[5] M. L. Allen, K. Jaakkola, K. Nummila, and H. Seppa, "Applicability of metallic nanoparticle inks in RFID applications," IEEE Transactions on Components and Packaging Technologies, vol. 32, no. 2, pp. 325-332, 2009.

[6] S. H. Yoon, J. H. Lee, P. C. Lee et al., "Sintering and consolidation of silver nanoparticles printed on polyimide substrate films," Macromolecular Research, vol. 17, no. 8, pp. 568-574, 2009.

[7] J. B. Szczech, C. M. Megaridis, D. R. Gamota, and J. Zhang, "Fine-line conductor manufacturing using drop-on-demand PZT printing technology," IEEE Transactions on Electronics $\mathrm{Pa}$ ckaging Manufacturing, vol. 25, no. 1, pp. 26-33, 2002.

[8] D. Kim and J. Moon, "Highly conductive ink jet printed films of nanosilver particles for printable electronics," Electrochemical and Solid-State Letters, vol. 8, no. 11, pp. J30-J33, 2005.

[9] R. D. Mancosu, J. A. Q. Quintero, and R. E. S. Azevedo, "Sintering, in different temperatures, of traces of silver printed in flexible surfaces," in Proceedings of the 11th International Conference on Thermal, Mechanical and Multi-Physics Simulation, and Experiments in Microelectronics and Microsystems (EuroSimE '10), pp. 1-5, Bordeaux, France, April 2010.

[10] "Samsung And Nokia Are Moving Towards With Flexible Screens Smartphone," http://www.mobiussite.com/samsung-nokia-moving-flexible/1830.html.

[11] "Samsung flexible display phones \& tablets in 2012," http:// www.slashgear.com/samsung-flexible-display-phones-tabletsin-2012-28191673/.

[12] Y. Min, "Mobile Terminal Using Flexible Display And Method Of Controlling The Mobile Terminal," U.S. Patent US 2010/ $0117975 \mathrm{Al}, 2010$.

[13] A. L. Scholtz and R. Weigel, "Antenna design for future multistandard and multi-frequency RFID systems," 2009.

[14] Youbok Lee, "Antenna circuit design for RFID applications," Microchip, pp. 1-50, 2003.

[15] M. Gebhart and R. Szoncso, "Optimizing design of smaller antennas for proximity transponders," in Proceedings of the 2nd International Workshop on Near Field Communication (NFC '10), pp. 77-82, Monaco, April 2010.

[16] C. R. Paul, Inductance-Loop and Partial, John Wiley \& Sons, New York, NY, USA, 2010.

[17] “Dimatix. Fujifilm," http://www.dimatix.com/.

[18] A. Rida, L. Yang, R. Vyas, and M. M. Tentzeris, "Conductive inkjet-printed antennas on flexible low-cost paper-based substrates for RFID and WSN applications," IEEE Antennas and Propagation Magazine, vol. 51, no. 3, pp. 13-23, 2009.

[19] U. Karthaus and M. Fischer, "Fully integrated passive UHF RFID transponder IC with 16.7- $\mu \mathrm{W}$ minimum RF input power," IEEE Journal of Solid-State Circuits, vol. 38, no. 10, pp. 1602-1608, 2003.
[20] Identification cards-Physical Characteristics, International Standard ISO 7810. 

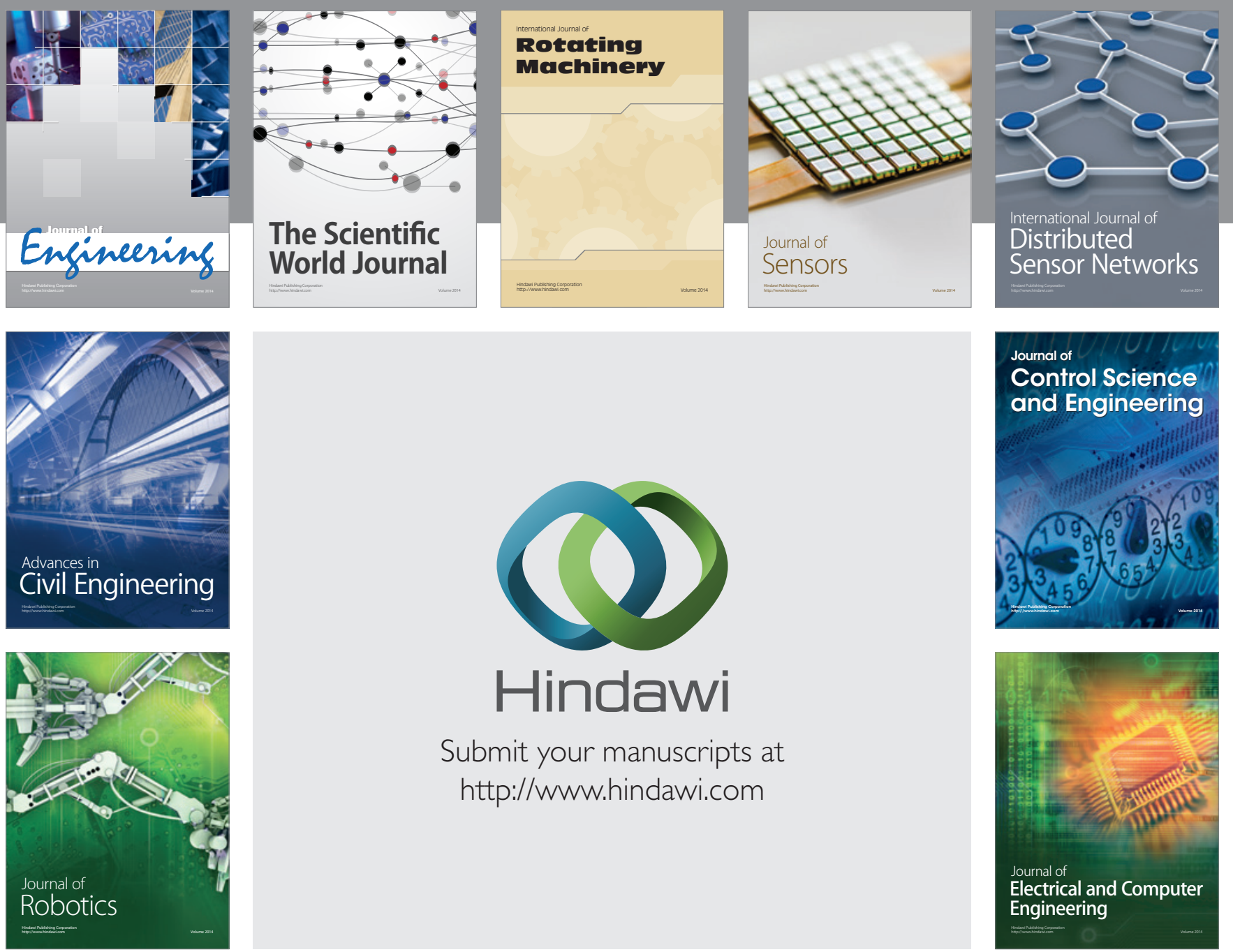

Submit your manuscripts at

http://www.hindawi.com
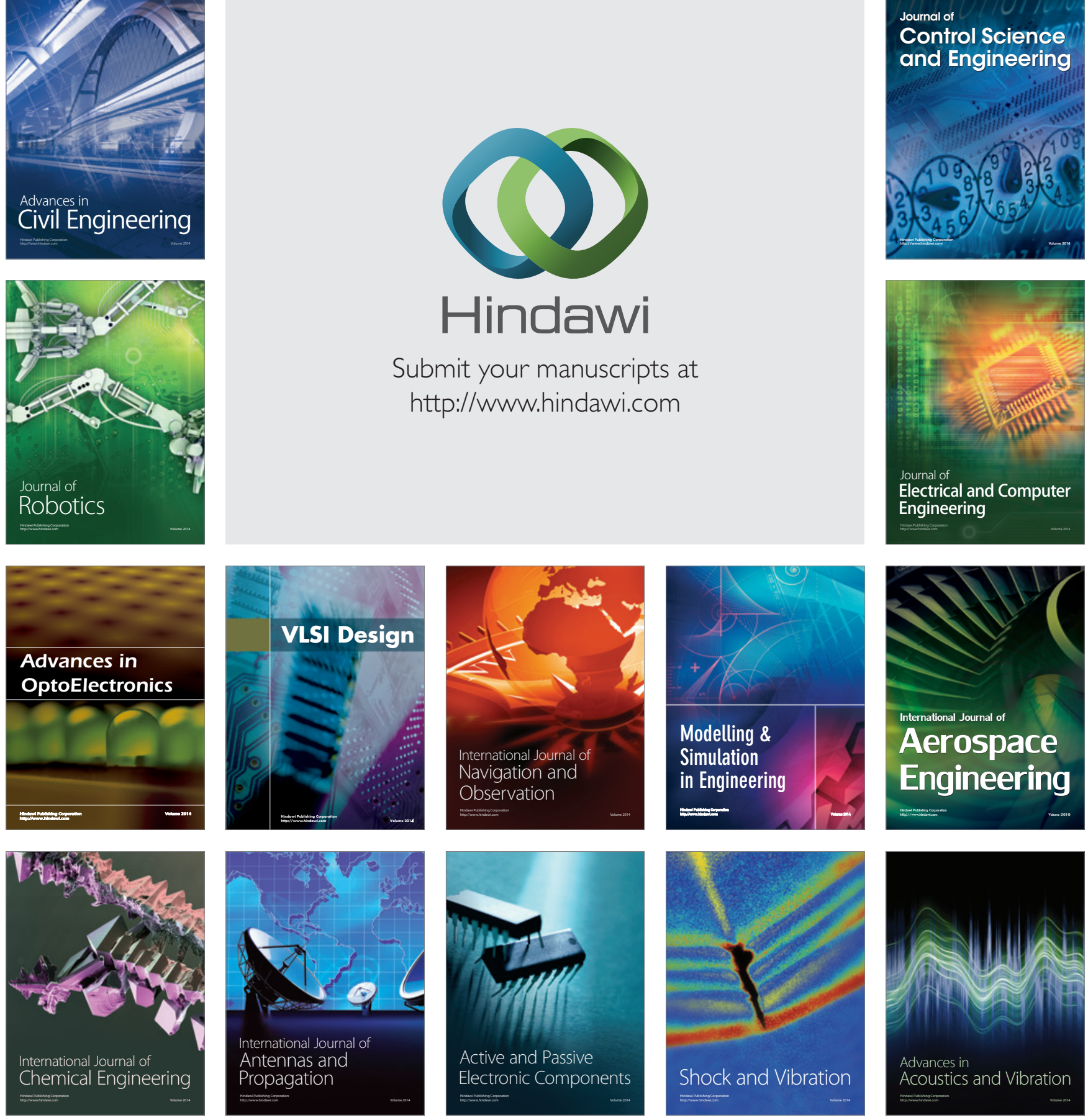